\title{
Contribución arqueométrica al estudio de las
} técnicas y de la organización de la producción de cerámica ritual en la sociedad Mochica (150-850 d.C., costa norte del Perú)

Contribution archéométrique à l'étude des techniques et de l'organisation de la production de céramique rituelle dans la société Mochica (150-850 ap. J.-C., côte nord du Pérou)

Archaeometric contribution to the study of ritual ceramic production techniques and organization in the Moche society (150-850 A.D., north coastal Peru)

\section{Agnès Rohfritsch}

\section{(c) OpenEdition \\ Journals}

Edición electrónica

URL: http://journals.openedition.org/bifea/2017

DOI: $10.4000 /$ bifea.2017

ISSN: 2076-5827

Editor

Institut Français d'Études Andines

Edición impresa

Fecha de publicación: 1 agosto 2010

Paginación: 389-412

ISSN: 0303-7495

\section{Referencia electrónica}

Agnès Rohfritsch, «Contribución arqueométrica al estudio de las técnicas y de la organización de la producción de cerámica ritual en la sociedad Mochica (150-850 d.C., costa norte del Perú) », Bulletin de l'Institut français d'études andines [En línea], 39 (2) | 2010, Publicado el 01 febrero 2011, consultado el 07 noviembre 2020. URL : http://journals.openedition.org/bifea/2017 ; DOI : https://doi.org/ 10.4000/bifea.2017

\section{(c) $(\mathrm{B})$}

Les contenus du Bulletin de l'Institut français d'études andines sont mis à disposition selon les termes de la licence Creative Commons Attribution - Pas d'Utilisation Commerciale - Pas de Modification 4.0 International. 


\title{
Contribución arqueométrica al estudio de las técnicas y de la organización de la producción de cerámica ritual en la sociedad Mochica (150-850 d.C., costa norte del Perú)
}

\author{
Agnès Rohfritsch*
}

\begin{abstract}
Resumen
Este artículo presenta los primeros resultados del estudio tecnológico de cerámica ritual mochica por medio de análisis de laboratorio. Esta investigación subraya la importancia de referirse, en la interpretación de los datos arqueométricos, a la información disponible sobre los contextos de producción antiguos (talleres), así como a los datos etnográficos. Por otra parte, tiende a demostrar que la cerámica ritual mochica, lejos de ser el resultado de una concepción técnica única, pudo ser producida a partir de varios procesos técnicos, pero con un mismo afán estético.
\end{abstract}

Palabras clave: cerámica, técnicas de fabricación, arqueometría, Mochica, Perú

Contribution archéométrique à l'étude des techniques et de l'organisation de la production de céramique rituelle dans la société Mochica (150-850 ap. J.-C., côte nord du Pérou)

\section{Résumé}

Dans cet article, sont présentés les premiers résultats de l'étude technologique de céramiques rituelles mochicas par le biais de méthodes de laboratoire. Cette recherche souligne la nécessité de prendre en compte, dans l'interprétation des données archéométriques, les informations disponibles dans le

Doctorante. Institut de Recherche sur les Archéomatériaux - Centre de Recherche en Physique Appliquée à l'Archéologie (UMR 5060, CNRS - Univ. Bordeaux 3), Pessac - France. E-mail: agnesrohfritsch@gmail.com 
répertoire archéologique (fouilles d'ateliers), ainsi que celles fournies par le répertoire ethnographique. D'autre part, elle tend à démontrer que la céramique rituelle mochica, loin d'être le fruit d'une conception technique unique, a pu être produite à partir de procédés techniques variés visant à un objet identique sur le plan esthétique.

Mots clés : céramique, techniques de fabrication, archéométrie, Mochica, Pérou

\title{
Archaeometric contribution to the study of ritual ceramic production techniques and organization in the Moche society (150-850 A.D., north coastal Peru)
}

\begin{abstract}
In this article, we will discuss the first results of a technological study of Moche ritual ceramics by the means of laboratory methods. This research underlines the importance of taking into account the information available in the archaeological record (excavation of workshops), and those provided by the ethnographic survey in the interpretation of the archeometric data. In addition, this work tends to show that Moche ritual ceramics, far from being the result of a single technical concept, could be produced through different technical processes, often leading, from an aesthetic point of view, to similar results.
\end{abstract}

Key words: ceramic, fabrication techniques, archaeometry, Moche, Peru

\section{INTRODUCCIÓN}

La costa norte del Perú entregó numerosos vestigios cerámicos que testimonian de la larga y compleja ocupación de esta región durante el periodo Mochica. El interés provocado por estos objetos se orientó, en primer lugar, hacia aspectos estilísticos, cronológicos e iconográficos, lo que permitió definir los grandes rasgos de esta cultura, su secuencia de ocupación1, así como su sistema simbólico. Actualmente, el estudio de la producción cerámica y de la producción de objetos artesanales en general, se abre a nuevas perspectivas con la aparición de nuevos interrogantes sobre la organización de esta sociedad compleja. Desde los años 1990, se sabe que la sociedad mochica no era organizada alrededor de un centro político único, sino que hubiera sido compuesta, en realidad, por varias organizaciones políticas independientes (Castillo \& Donnan, 1994; Castillo \& Uceda, 2008). En este marco, el estudio de la producción de ciertos objetos puede ser una herramienta poderosa para identificar las características técnicas de los diferentes grupos que formaron esta sociedad, especialmente en el caso de los objetos ceremoniales, que tienen una implicación directa en el proceso

1 Gracias a los estudios llevados a cabo, dos secuencias cronológicas fueron propuestas: una secuencia en cinco fases (Mochica I a V) para el territorio Mochica Sur (Larco, 1948), y una secuencia en tres fases (Mochica Temprano, Medio y Tardío) para el territorio Mochica Norte (Castillo \& Donnan, 1994). 
de materialización de la ideología de una elite (Peregrine, 1991; DeMarrais et al., 1996). En esta perspectiva, enfocaremos nuestro discurso sobre las cerámicas definidas como «rituales», definir cuál función está directamente relacionada con la práctica de ceremonias religiosas y/o funerarias, en contraste con las cerámicas «domésticas» que son utilizadas en las actividades de la vida cotidiana.

En este artículo, examinaremos, en primer lugar, los datos proporcionados por la excavación de contextos de producción de cerámica mochica y por los estudios etnográficos de comunidades alfareras actuales. En segundo lugar, expondremos los primeros resultados del estudio tecnológico de cerámicas mochicas de los valles de Jequetepeque y Chicama por medio de unos análisis arqueométricos.

\section{LOS CONTEXTOS DE PRODUCCIÓN}

El primer descubrimiento de un taller de producción de cerámica mochica fue identificada por Bawden en el sitio de Galindo (Bawden, 1982; 1996: 97-101). Las excavaciones revelaron un taller de pequeño tamaño que funcionó durante la fase Mochica V. Los objetos producidos son de diversos tipos: figurinas, cántaros con cara-gollete, botellas de doble cuerpo, vasijas templadas para almacenar y cocinar, etc... Según Bawden, estas vasijas se cocían en un pozo abierto que producía una atmósfera de cocción parcialmente reducida, antes de ser empleadas como objetos utilitarios en los alrededores directos del sitio. Sin embargo, el autor considera también un posible comercio a más larga distancia. En cuanto a la identidad de los artesanos que producían estos objetos, parece que su posición social haya sido poco elevada y nada nos lleva a pensar que su trabajo estaba supervisado o controlado por autoridades gobernantes (Bawden, 1982).

En el sitio de las huacas de Moche, las primeras evidencias de un taller de producción de cerámica utilitaria fueron descubiertas en las laderas del Cerro Blanco (Topic, 1977). A estas primeras evidencias se agregaron los talleres alfareros identificados al principio de los años 1990 en la zona urbana (Armas et al., 1993; Uceda \& Armas, 1997). Estos talleres, que se sitúan a aproximadamente a $150 \mathrm{~m}$ de la Plataforma I de la huaca de la Luna, se componen de tres niveles de ocupación, todos pertenecientes a la fase Mochica IV. Los autores señalan la presencia de zonas de combustión, moldes y matrices de moldes, cerámica cruda, cocida o deformada por la cocción, metates y manos de moler, y grandes tinajas para almacenar, lo que no deja ninguna duda sobre la función de este espacio. Como en el taller de Galindo, una amplia gama de objetos era producida (figurinas, silbatos, sonajeros, ocarinas, jarras, crisoles, aplicaciones, pendientes, piruros, etc.), pero en contraste con este último, la gran mayoría de los objetos producidos en el taller de huacas de Moche son de uso ritual.

En Pampa Grande, en el valle de Lambayeque-La Leche, Shimada (1994a) identificó algunas evidencias de producción cerámica que correspondían a la fase Mochica V, entre ellas fragmentos de moldes, pulidores de piedra, ollas aparentemente sin uso y huellas de combustión (cenizas y carbones). Las formas producidas eran diversas (platos, tazones, floreros, ollas de cuello estrecho, botellas de asa estribo 
y discos perforados) y se caracterizan por una cocción en atmósfera reductora. Shimada, basándose en la limitada distribución y cantidad de cerámicas de pasta negra (reducida) en Pampa Grande, sugiere que la producción de este taller respondía solamente a la demanda del sitio.

En el valle de Chicama, Glenn Russel y su equipo (Russel et al., 1994; Russel \& Jackson, 2001) excavaron un amplio taller que se extiendía sobre una superficie de aproximadamente $9000 \mathrm{~m}^{2}$, con una densa zona de desechos en el centro (aprox. $5000 \mathrm{~m}^{2}$ ). Este taller funcionó durante la fase Mochica IV y corresponde, según los fechados realizados por C14 (Attarian, 1996: 15-16), a una sola fase de ocupación. Las estructuras de cocción identificadas son de tipo pozo abierto y mostraban a veces evidencias de muros de dos o tres hileras de adobes que circundaban la estructura. También se encontró lo que los autores llamaron un «horno de simple cámara», pero la descripción de este quedó muy somera (Russel \& Jackson, 2001: 164). Una vez más, los objetos producidos eran de varios tipos: instrumentos de música, piruros, figurinas, floreros, cántaros, cuencos, ollas de cuello evertido, jarras, ralladores, botellas de un solo gollete, etc. Sin embargo, como en el taller de la zona urbana de huacas de Moche, la gran mayoría de los objetos producidos parecen de uso ritual (Russel \& Jackson, 2001: 165) y la proximidad de este taller con el sitio de Mocollope, un importante centro cívico y ceremonial mochica que se sitúa aproximadamente a 1,5 km, sugiere que la producción de Cerro Mayal respondía a la demanda de los habitantes y visitantes de Mocollope (Russel et al., 1994: 206).

Por otra parte, la presencia de un área residencial adyacente al taller y en donde se encontraron moldes, lleva a pensar que Cerro Mayal constituía un amplio conjunto en el cual los alfareros vivían y trabajaban (Russel et al., 1994: 209; Russel \& Jackson, 2001: 165).

Por último, en el valle de Santa, Wilson menciona la presencia de moldes en un posible taller alfarero de la fase Mochica IV en Pampa de los Incas (Wilson, 1988: 211).

Las excavaciones en estos diversos contextos de producción no nos permiten reconstituir en su totalidad la cadena operatoria de la producción cerámica en la época mochica; sin embargo nos proporcionan cierta información. El cuadro siguiente presenta los diferentes elementos encontrados en estos talleres con respecto a las diferentes etapas de fabricación de los objetos (cuadro 1).

Sobre la primera etapa, que corresponde a la preparación de la pasta, no disponemos de mucha información. Sin embargo, la presencia de metates y manos de moler en el taller de las huacas de Moche indica el molido de las materias primas (de la arcilla y/o del temperante). Por otra parte, los fragmentos de arcilla cruda descubiertos en este taller, y su comparación por activación neutrónica con arcillas procedentes de los alrededores del sitio, indican que estas materias primas son de procedencia local (Chapdelaine et al., 1995; 2001).

La etapa de fabricación más documentada por la excavación de contextos de producción es sin duda la elaboración de las vasijas y su decoración. Se nota 
Cuadro 1 - Síntesis de las diferentes herramientas y estructuras encontradas en talleres de producción de cerámica mochica en relación con las diferentes etapas de la cadena operatoria

\begin{tabular}{|c|c|c|c|c|}
\hline \multirow{2}{*}{ Taller } & \multirow{2}{*}{$\begin{array}{l}\text { Preparación de } \\
\text { la pasta }\end{array}$} & \multirow{2}{*}{$\begin{array}{c}\text { Elaboración y } \\
\text { decoración }\end{array}$} & \multicolumn{2}{|c|}{ Cocción } \\
\hline & & & Estructuras & Combustible \\
\hline $\begin{array}{l}\text { Huacas de } \\
\text { Moche } \\
\text { (Armas et al., } \\
\text { 1993; Uceda \& } \\
\text { Armas, 1997) }\end{array}$ & $\begin{array}{l}\text { - metates y } \\
\text { manos de moler } \\
\text { - grandes tinajas } \\
\text { (para almacenar } \\
\text { agua ?) }\end{array}$ & $\begin{array}{l}\text { - moldes y matrices de } \\
\text { moldes } \\
\text { - plato de alfarero (?) } \\
\text { - pulidores de piedra y } \\
\text { alisadores de madera. }\end{array}$ & $\begin{array}{l}\text { - pozo } \\
\text { abierto }\end{array}$ & \\
\hline $\begin{array}{l}\text { Pampa de los } \\
\text { Incas } \\
\text { (Willson, 1988: } \\
211 \text { ) } \\
\end{array}$ & & - moldes & & \\
\hline $\begin{array}{l}\text { Cerro Mayal } \\
\text { (Russel et al., } \\
\text { 1994; Russel \& } \\
\text { Jackson, 2001) }\end{array}$ & & $\begin{array}{l}\text { - moldes } \\
\text { - pulidores }\end{array}$ & $\begin{array}{l}\text { - pozo } \\
\text { abierto } \\
\text { - horno } \\
\text { de simple } \\
\text { cámara } \\
\end{array}$ & $\begin{array}{l}\text { - algarrobo } \\
\text { - cola de } \\
\text { caballo }\end{array}$ \\
\hline $\begin{array}{l}\text { Galindo } \\
\text { (Bawden, 1982; } \\
\text { 1996: 97-101) }\end{array}$ & & - moldes & $\begin{array}{l}\text { - pozo } \\
\text { abierto }\end{array}$ & $\begin{array}{l}\text { - leña } \\
\text { - estiércol } \\
\text { de llama }\end{array}$ \\
\hline
\end{tabular}

la presencia de moldes, o fragmentos de moldes, en todos los talleres, lo que confirma el uso extendido de esta técnica y su desarrollo durante la época Mochica (Donnan, 2004). Sin embargo, no significa que la elaboración de estos objetos se hacía sistemáticamente por moldeado. Técnicas como la compresión digital, el enrollado y el enchapado no pueden ser descartadas. Desgraciadamente, estas técnicas, que generalmente no necesitan ninguna herramienta específica, dejan pocas huellas en el registro arqueológico.

Además de ser utilizados para confeccionar el cuerpo de las vasijas, los moldes también podían ser usados para decorar parte de la vasija, como en el caso de los cántaros con cara-gollete (una buena ilustración de este proceso puede consultarse en Anders et al., 1994: 257, fig. 10).

En el taller de las huacas de Moche, los investigadores encontraron un objeto singular que fue interpretado como un plato de alfarero. Pero la forma de este objeto, que corresponde a un plato semicircular perforado de dos huecos, se aleja bastante de la forma tradicional de los platos de alfarero (plato circular de superficie plana o cóncava y base convexa para permitir la rotación), lo que hace problemática la interpretación de este objeto. En Pampa Grande, Shimada también encontró platos de cerámica negra con base ligeramente convexa que interpretó como posibles platos de alfarero (Shimada, 1994a: 194-195). En este caso, la interpretación parece más convincente, aunque el autor no señala ninguna 
evidencia del uso de estos objetos como platos de alfarero, como por ejemplo el deterioro de la base por la rotación.

En lo que se refiere a la última etapa de fabricación de los objetos, es decir la cocción, la mayoría de los contextos de producción de cerámica de época mochica atestiguan del empleo de formas de cocción generalmente calificadas de «primitivas», como la cocción en pozo abierto. Este tipo de estructura de cocción fue encontrado en Cerro Mayal, en las huacas de Moche y en Galindo. Sin embargo, nos parece importante señalar que las excavaciones conducidas por Shimada en Batán Grande (Shimada et al., 1994) demuestran que la cocción en horno era conocida y empleada en el periodo Formativo2. No se puede excluir, entonces, que los alfareros mochicas hayan empleado también este tipo de cocción. Como lo hemos visto previamente, Russel \& Jackson (2001) mencionan la presencia de un horno de cámara entre las estructuras de cocción descubiertas en Cerro Mayal, pero sin dar más precisiones sobre el funcionamiento de esta estructura.

Una de las principales ventajas de la cocción en horno, en comparación con la cocción en área abierta o en pozo, es un mejor control de la atmósfera durante el proceso de cocción. Con respecto a este tema, la hipótesis de una solución intermedia entre la cocción en pozo abierto y la cocción en horno que proponen Anders et al. (1994: 261-262) acerca de la elaboración de cerámicas del Horizonte Medio en Maymi es particularmente interesante. En este taller, donde las estructuras de cocción descubiertas corresponden a pozos de combustión que miden aproximadamente $3 \mathrm{~m}$ de diámetro por $40 \mathrm{~cm}$ de profundidad, los autores consideran dos posibles formas de cocción. La primera, que podemos calificar de «clásica» para este tipo de estructura, consiste en colocar las vasijas directamente en contacto con el combustible (cocción mixta). La segunda, calificada de cocción indirecta o por «emuflado» (Anders et al., 1994: 261), consiste en colocar las vasijas primero y después cubrirlas completamente con grandes tiestos, lo que permite aislar las vasijas del combustible. La cocción se hace de manera progresiva, aumentando poco a poco la cantidad de combustible. Como lo subrayan los autores, esta técnica permite obtener un mejor control de la atmósfera de cocción y conduce a una oxidación perfecta de las vasijas (Anders et al., 1994: 262).

Por otra parte, los carbones encontrados en estas diversas estructuras de cocción nos informan sobre la naturaleza de los combustibles empleados. En Batán Grande y en Cerro Mayal (Shimada et al., 1994: 78; Russel et al., 1994: 211), el examen de los carbones de madera demostró el uso de algarrobo (Prosopis pallida), árbol o arbusto espinoso que, en el Perú, se encuentra en los desiertos de la costa norte

2 En Batán Grande, en el valle de Lambayeque-La Leche, Shimada encontró 57 hornos de alfareros, repartidos sobre cuatro niveles de ocupación. Se trata de hornos semisubterráneos que se componen de un espacio donde se colocan las cerámicas (cámara de cocción), y de un espacio para el combustible (fogón). La cámara de cocción está cubierta por una bóveda de arcilla y tiene una apertura en su extremidad que sirve como chimenea. Las experimentaciones de cocción efectuadas por Shimada y su equipo han demostrado que estos hornos permiten alcanzar una temperatura máxima de más o menos $800^{\circ} \mathrm{C}$ (Shimada et al., 1994: 102). 
entre 0 y 1500 m de altura. También llamado «árbol multifunción», el algarrobo es todavía usado hoy en día. Considerado como un combustible de muy buena calidad, su madera muy resistente hace también de él un excelente material de construcción (Moutarde, 2006: 126).

En el sitio de Cerro Mayal, Russel et al. (1994: 211) identificaron también una planta conocida como «cola de caballo» (Equisetum giganteum). Anders et al. (1994: 262) han postulado igualmente su utilización en el sitio de Maymi. Según estos autores, esta planta podría haber servido como combustible de rápida quema durante la última etapa de lo que describieron como cocción indirecta, a fin de obtener una atmósfera perfectamente oxidante, evitando así la formación de manchas por una mala oxidación de las paredes.

En el taller de Galindo, Bawden señaló la presencia, además de leña, de una gran cantidad de estiércol de llama y postuló que se utilizaba como combustible para la cocción de las vasijas (Bawden, 1996: 98).

Tratándose de la organización de la producción, podemos constatar que todos estos talleres se caracterizan por la fabricación de una amplia gama de objetos de cerámica. Ninguno, en efecto, parece testimoniar de una producción especializada dedicada a la fabricación de un tipo particular de objeto o de forma. Sin embargo, en Cerro Mayal y en la zona urbana de las huacas de Moche, observamos que la mayoría de los objetos producidos eran de uso ritual (instrumentos de música, figurinas, platos para el servicio ritual, etc.), aunque también se producía en estos talleres cerámicas de uso doméstico (ollas, jarras, cántaros, vasijas templadas para almacenar y cocinar, platos, ralladores, etc.). Esta fuerte proporción de objetos rituales sugiere que la producción de estos dos talleres era destinada a responder a la demanda generada por las actividades rituales que se desarrollaban en los dos centros administrativos y religiosos localizados en los alrededores. El taller de las huacas de Moche, está ubicado a solo unos metros de una de las principales estructuras ceremoniales del sitio (la huaca de la Luna). Esta proximidad constituye, en la opinión de algunos, una indicación clara de una supervisión de la producción por altos dirigentes mochicas, considerando así los alfareros que trabajaban en este taller como artesanos especializados afiliados a la elite gobernante (Rengifo \& Rojas, 2008: 335 ; Bernier, 2008: 43). En el caso de Cerro Mayal, en cambio, el taller se encuentra un poco al margen del centro ceremonial asociado (Mocollope). Ni el área del taller, ni el área residencial asociada presentan evidencia de ocupación por una elite o de función ceremonial, y ninguna estructura parece restringir el acceso al taller. Para Russel et al. (1994) aunque la supervisión de las elites pudiera haber desempeñado un papel en la producción de ciertas formas fabricadas en el taller, nada permitía afirmar que la producción haya sido directamente ligada a la clase gobernante bajo la forma de un taller dependiente (Russel et al., 1994: 221). 


\section{APORTES DE LA ETNOGRAFÍA AL ESTUDIO DE LA PRODUCCIÓN DE CERÁMICA PREHISPÁNICA}

Desde mucho tiempo atrás, arqueólogos y etnógrafos se han interesado en el estudio de comunidades alfareras actuales a fin de entender mejor la producción antigua. La mayoría de los trabajos realizados han versado sobre la costa norte, y especialmente la región de Piura (Camino, 1982; 1989; Sabogal Wiesse, 1982; Bankes, 1985; Monzon, 1991; Shimada, 1994b), la sierra central (Lavallée, 1967; Donnan, 1971; Arnold, 1972; 1975; Pozzi-Escot et al., 1993; Druc, 1996; 2000; Ramon Joffré, 1999) y la sierra sur (Tschopik, 1950). Los objetos producidos por estas comunidades son principalmente cerámicas utilitarias (ollas, cántaros, tinajas...) y las técnicas y los modos de producción empleados para su fabricación probablemente son muy diferentes de los que fueron utilizados para la fabricación de cerámica ritual durante la época prehispánica. Sin embargo, estas investigaciones tienen el mérito de subrayar la existencia de tradiciones cerámicas propias a ciertas regiones, como por ejemplo el uso extendido del paleteado en la costa norte, mientras que el plato de alfarero domina en las regiones sureñas (Ramon Joffré, 1999: 239-243; 2008). En cierta medida, esta repartición regional de los estilos técnicos parece ser un reflejo de tradiciones antiguas (Tschopik, 1950; Bankes, 1985).

Estos estudios etnográficos también demostraron la importancia de tomar en cuenta los aspectos ecológicos que influencian la producción cerámica (Shimada, 1985; Druc, 1996). La tecnología y los modos de producción en una sociedad no solo dependen de la forma de organización social y política, sino también de la influencia del medio ambiente. Tratándose de las técnicas de fabricación, varios estudios subrayan una diferencia, entre las comunidades serranas y costeñas, en el proceso de preparación de la pasta. La mezcla de arcillas muy plásticas con arcillas más arenosas para introducir temperante se encuentra con más frecuencia en las comunidades serranas (Litto, 1976; Ravines, 1978; 1989; Druc, 1996; Ramón Joffré, 1999), mientras que la mezcla de arcilla con arena es más común en la producción costeña (Litto, 1976; Ravines, 1978; Shimada, 1994b).

La influencia del medio ambiente se observa también al nivel de la organización de la producción. Como lo enfatiza Dean Arnold (Arnold, 1985: 90), el ambiente árido y la ausencia casi total de lluvia que caracterizan la costa norte peruana constituyen sin duda un factor determinante en la organización de la producción cerámica en esta región. En contraste con las zonas serranas, donde la alternación entre una temporada seca y una temporada húmeda obliga a los alfareros a dejar su actividad parte del año3, la aridez de la zona costanera brinda la oportunidad de producir vasijas a lo largo del año. Estas condiciones climáticas, entonces,

3 En Santo Domingo de los Olleros, en la sierra central, la temporada de lluvias (entre noviembre y abril) está marcada por importantes modificaciones en la organización de la comunidad, con la interrupción de la actividad alfarera y la partida de los ganaderos que bajan hacia la zona costeña (Ramón Joffré, 1999: 218). 
proporcionan un marco ideal para el desarrollo de una especialización cerámica a tiempo completo.

\section{EL ESTUDIO TECNOLÓGICO DE LOS OBJETOS PRODUCIDOS}

Como lo hemos visto más arriba, el estudio de los vestigios arqueológicos relacionados con la producción de cerámica es esencial para formarse una primera idea de la tecnología empleada y de la organización de esta producción. En cuanto al estudio de datos etnográficos en una perspectiva etnoarqueológica, aunque nos proporcione cierta información que complementa los datos arqueológicos, contribuye más bien a orientar los interrogantes arqueológicos que a resolverlos. Finalmente, el estudio tecnológico de los objetos producidos, por medio de métodos de laboratorio, tiene la ventaja de permitir un estudio más profundo de la naturaleza de los materiales empleados y de las técnicas que se usaron para transformarlos.

Este estudio técnico de cerámica ritual mochica por medio de métodos de laboratorio se está realizando actualmente. No pretendemos presentar de manera exhaustiva los resultados obtenidos, sino más bien señalar ciertos datos que parecieron particularmente interesantes desde el punto de vista tecnológico y que contribuyeron a orientar el desarrollo de este estudio.

\section{1. Material estudiado y métodos de análisis}

En este trabajo, presentaremos los primeros resultados de análisis arqueométricos conducidos a partir de fragmentos de cerámicas mochicas procedentes de los valles de Jequetepeque y Chicama. Estos fragmentos son de cerámicas rituales que se caracterizan por su fuerte valor simbólico. Un primer grupo de 12 muestras se compone de vasijas que pertenecen a las fases más antiguas de la secuencia de ocupación mochica. Abarca fragmentos de cerámica Mochica Temprano de Dos Cabezas y de cerámica Mochica I-II del sitio El Brujo (fig. 1). Estos fragmentos corresponden a botellas de asa estribo representando en tres dimensiones animales o seres humanos, así como creaturas antropomorfas o zoomorfas. Un segundo grupo abarca 24 muestras de cerámica Línea Fina procedente de San José de Moro y Pacatnamú (fig. 2). Estas vasijas, que son típicas del Mochica Tardío en el valle de Jequetepeque, son botellas de asa estribo adornadas con una decoración pintada compleja que representa de manera detallada diferentes ceremonias o actividades rituales de la cultura Mochica (Donnan \& McClelland, 1999).

La mayoría de las muestras analizadas fueron extraídas de cerámicas ya fragmentadas para las cuales era posible, sin embargo, identificar la forma original a partir de los fragmentos. El hecho de trabajar a partir de tiestos, y no de objetos completos, nos permitió librarnos de la obligación de recurrir a métodos de análisis no destructivos o de realizar muestras de muy pequeñas dimensiones, las cuales, en el caso de la cerámica, pueden plantear problemas de representatividad de la muestra con respecto al objeto del cual proviene. 
Agnès Rohfritsch

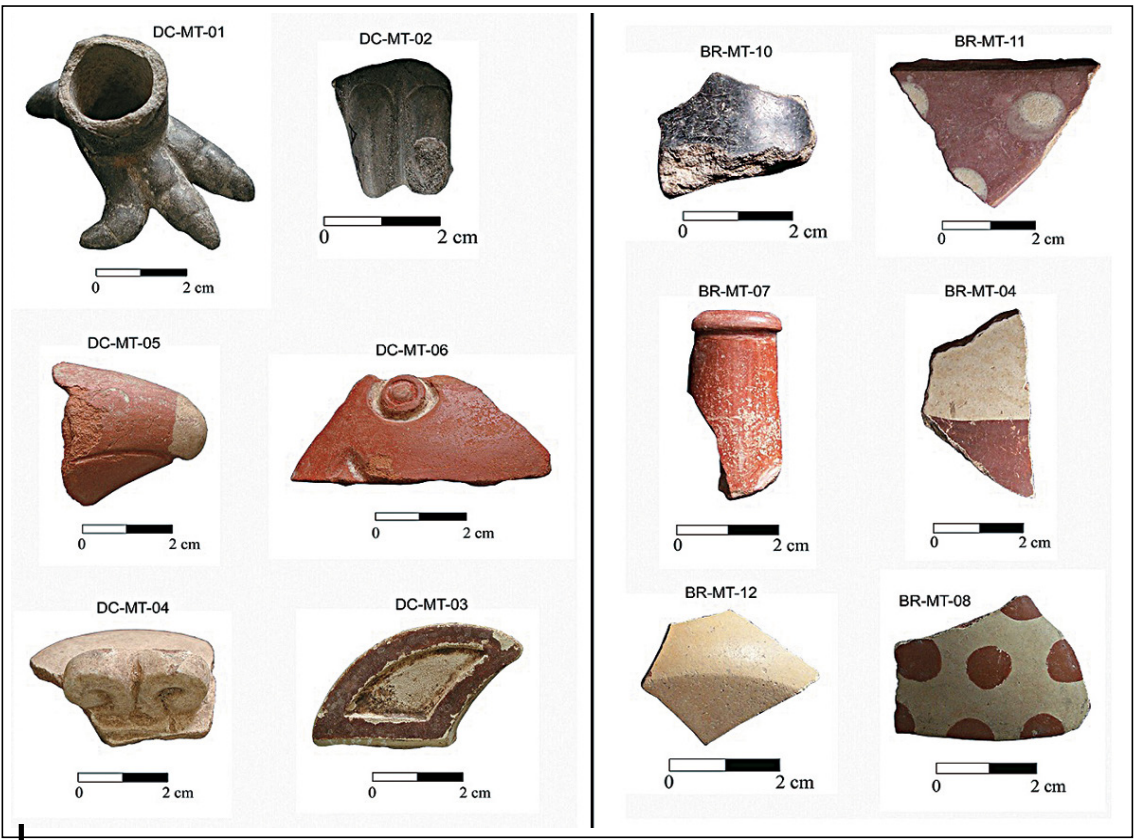

Figura 1 - Fragmentos de cerámica Mochica Temprano de Dos Cabezas (izquierda) y de cerámica Mochica I-II de El Brujo (derecha)

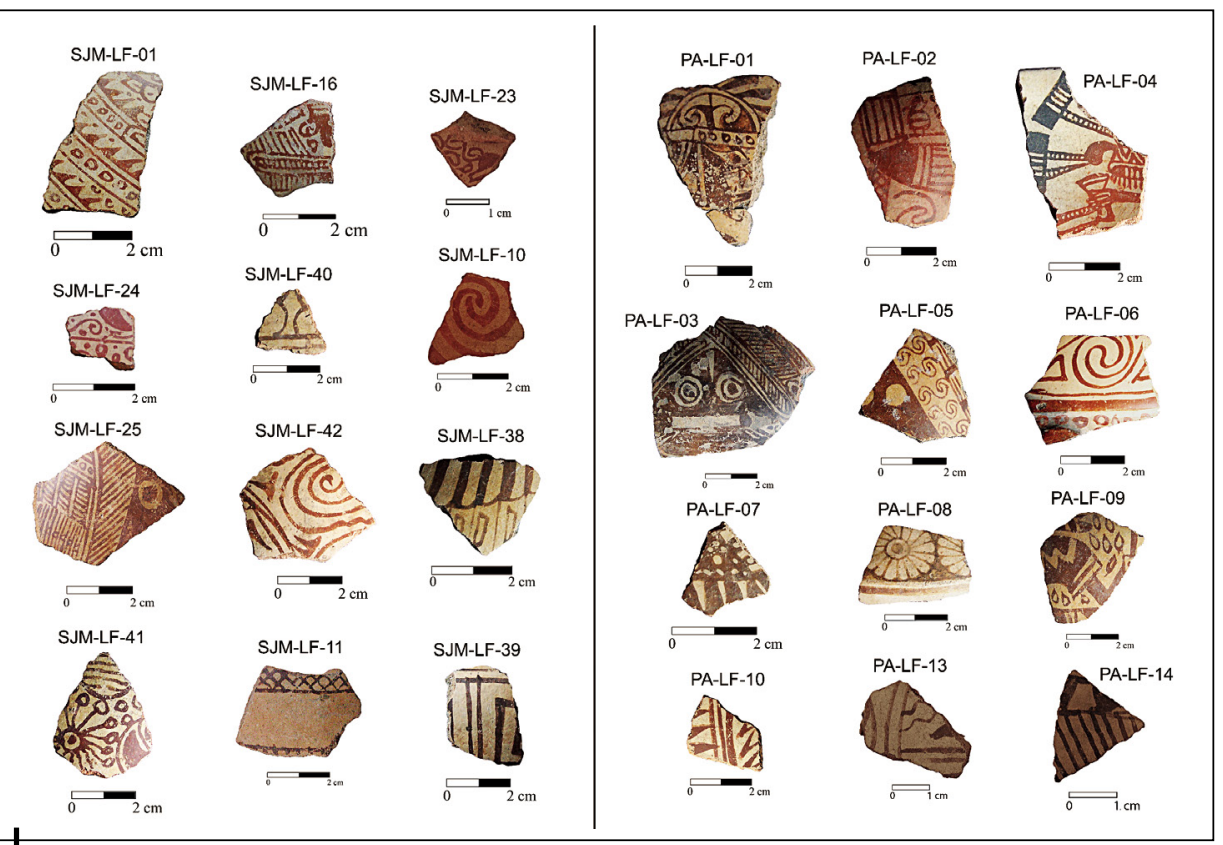

Figura 2 - Fragmentos de cerámica Mochica Tardío tipo Línea Fina procedentes de San José de Moro (izquierda) y de Pacatnamú (derecha) 
Los métodos de análisis utilizados incluyen observaciones en microscopia óptica de secciones pulidas para caracterizar la textura de las pastas (color y porosidad, formas, dimensiones, composición mineral de las arcillas y repartición del temperante, técnicas de decoración). Estas fueron complementadas por observaciones con un microscopio electrónico de barrido (MEB), acoplado a un sistema de espectrometría de rayos X en dispersión de energía (EDS) para efectuar también unos análisis semicuantitativos de las concentraciones de elementos mayores y menores de los diferentes constituyentes de las cerámicas (pasta, engobe, pintura4).

La difracción de rayos $X$ fue utilizada para la determinación de la composición mineralógica de las muestras, que nos proporcionaba informaciones sobre las técnicas de cocción. En efecto, se sabe que, durante la cocción de una cerámica, se producen transformaciones de fases cristalinas bajo el efecto del calor (Périnet, 1960; Peters \& Iberg, 1978; Maggetti, 1982). El estudio de estas fases cristalinas permite, entonces, estimar a qué temperatura fue sometida una vasija. Generalmente se trata de la «temperatura de cocción equivalente», es decir la temperatura de cocción que, mantenida durante una hora, habría producido sobre una cerámica cruda efectos similares a los que se observan sobre el tiesto que se analiza (Picon, 1973; Tite, 1999). Los datos sobre la composición cristalográfica fueron complementados por observaciones en microscopia electrónica de barrido a importantes aumentos (x900 y x2000) a fin de observar la microestructura de la matriz arcillosa. De hecho, las transformaciones de esta microestructura durante la cocción, con una vitrificación progresiva de la fracción arcillosa y una modificación de la porosidad, proporcionan también información sobre las temperaturas de cocción (Maniatis \& Tite, 1981).

Sin embargo, hay que recordar que la temperatura alcanzada no es el único aspecto que permite explicar el resultado obtenido. La naturaleza de los materiales empleados (composición mineralógica y química), la granulometría de los componentes, la curva de subida de temperatura, así como el cambio de atmósferas durante la cocción también son factores sumamente importantes (Echallier, 1984).

\section{2. Primeros resultados}

Las observaciones de 36 secciones revelan, en la mayoría de las muestras, un temperante fino (partículas inferiores a $500 \mu \mathrm{m}$ ) y abundante (entre 20 y $40 \%$ ).

4 Para describir las diferentes capas de decoración de las vasijas de este estudio, hemos seguido la terminología propuesta por M. Picon (Picon, 1973) para la distinción entre engobe y pintura. Este autor propone diferenciar estos dos elementos no por la naturaleza del material empleado (barbotina/pigmentos), sino por su utilización en la organización del decorado. Entonces, hablaremos de engobe para designar una capa que cubre la totalidad de la vasija, formando así el fondo de la decoración. En cambio, hablaremos de pintura para las capas que constituyen el motivo de esta decoración. 
Este temperante es únicamente de naturaleza mineral (no temperante vegetal) y se compone principalmente de cuarzos y feldespatos.

Sobre los colores de pasta, las cerámicas del periodo inicial pueden dividirse en tres grupos: las cerámicas de pasta negra, las cerámicas de pasta roja y las de pasta blanca (fig. 3). El análisis químico (EDS) de la fracción fina (matriz arcillosa) indica una concentración de calcio $(\mathrm{CaO})$ mucho más elevada en las cerámicas de pasta blanca (aprox. 20 \%), lo que sugiere el uso de arcillas calcáreas para la fabricación de estas vasijas (fig. 4).
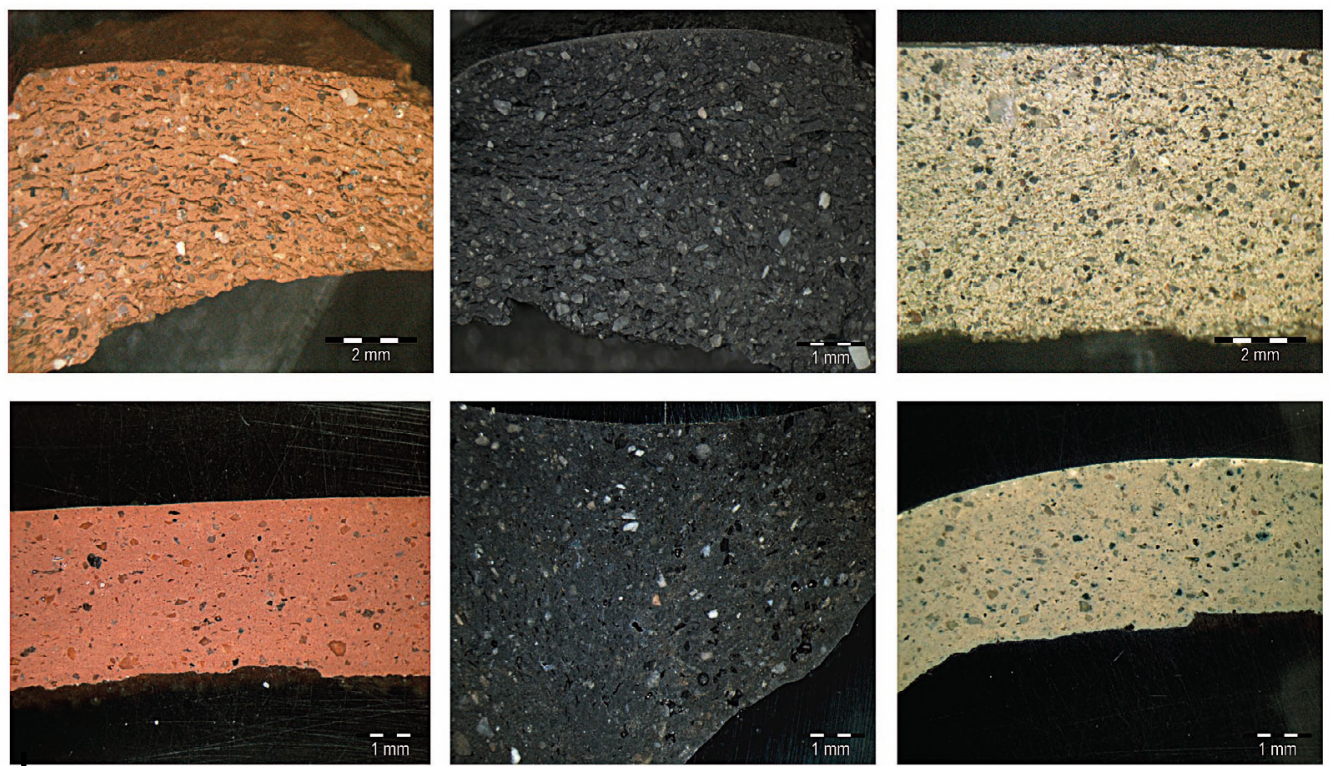

Figura 3 - Observaciones en microscopia óptica de las secciones de vasijas de pasta roja, negra y blanca de Dos Cabezas (arriba) y El Brujo (abajo)

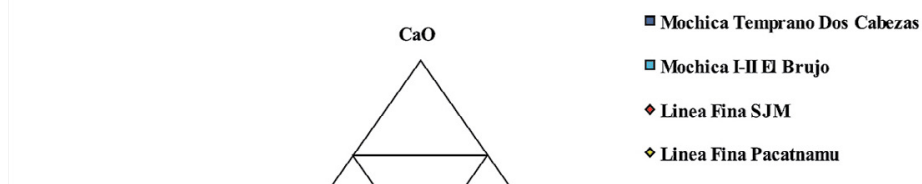

$\mathrm{Fe} 2 \mathrm{O3}$

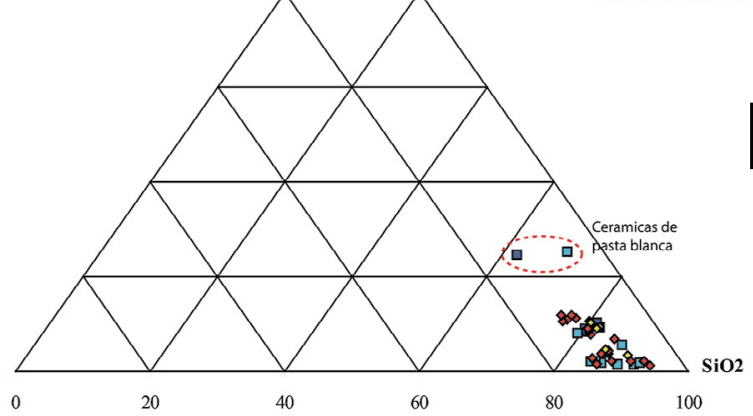

Figura 4 - Diagrama ternario de las concentraciones de calcio $(\mathrm{CaO})$, hierro $\left(\mathrm{Fe}_{2} \mathrm{O}_{3}\right)$ y silicio $\left(\mathrm{SiO}_{2}\right)$ de las muestras analizadas

Se nota una proporción de $\mathrm{CaO}$ mucho más alta para los dos fragmentos de cerámica de pasta blanca de Dos Cabezas (DC-MT-03) y El Brujo (BRMT-12) 
Desde el punto de vista tecnológico, las cerámicas calcáreas son muy interesantes porque su cocción solo se puede hacer a baja temperatura o, al contrario, a una temperatura bastante alta (Picon, 1992: 117). Eso proviene de que las arcillas calcáreas contienen carbonatos de calcio que, a partir de $650-700^{\circ} \mathrm{C}$, empiezan a descomponerse para dar lugar a la formación de cal (Peters \& Iberg, 1978 ; Maggetti, 1982). Si la cocción sigue hasta una temperatura suficiente, la cal se recombina con los componentes de la arcilla para formar aluminosilicatos de calcio o silicatos de calcio/ magnesio que quedan estables después de la cocción. Si, al contrario, la cocción no sigue hasta una temperatura suficiente, la cal se retransforma en carbonato de calcio, un fenómeno que se acompaña de un fuerte aumento de volumen, lo cual puede ocasionar daños a las vasijas. En el caso de una cocción a muy baja temperatura, no existe ningún riesgo puesto que la calcita original no se descompone.

Ahora bien, los dos fragmentos de cerámica de pasta blanca de Dos Cabezas y El Brujo presentan las características de una cocción a alta temperatura. Los resultados del análisis por difracción de rayos $\mathrm{X}$ de la muestra DC-MT-03 indica la presencia de fases minerales que se forman a alta temperatura como el diópsido, que empieza a formarse a partir de $800-850^{\circ} \mathrm{C}$, y la wollastonita, que aparece a partir de $850-900^{\circ} \mathrm{C}$ (Peters \& Iberg, 1978). Se nota también la presencia de feldespatos tipo plagioclasas que podrían corresponder a la anortita, otro mineral que se forma a alta temperatura en las arcillas calcáreas. La gehlenita, en cambio, no es detectada, lo que podría indicar una temperatura superior a $950^{\circ} \mathrm{C}$, temperatura a partir de la cual este mineral se descompone (fig. 5). Además, la observación de la microestructura de estas dos muestras en MEB (fig. 6) muestra un nivel de vitrificación de las pastas que sugiere una temperatura al menos superior a $850^{\circ} \mathrm{C}$ (Maniatis \& Tite, 1981).

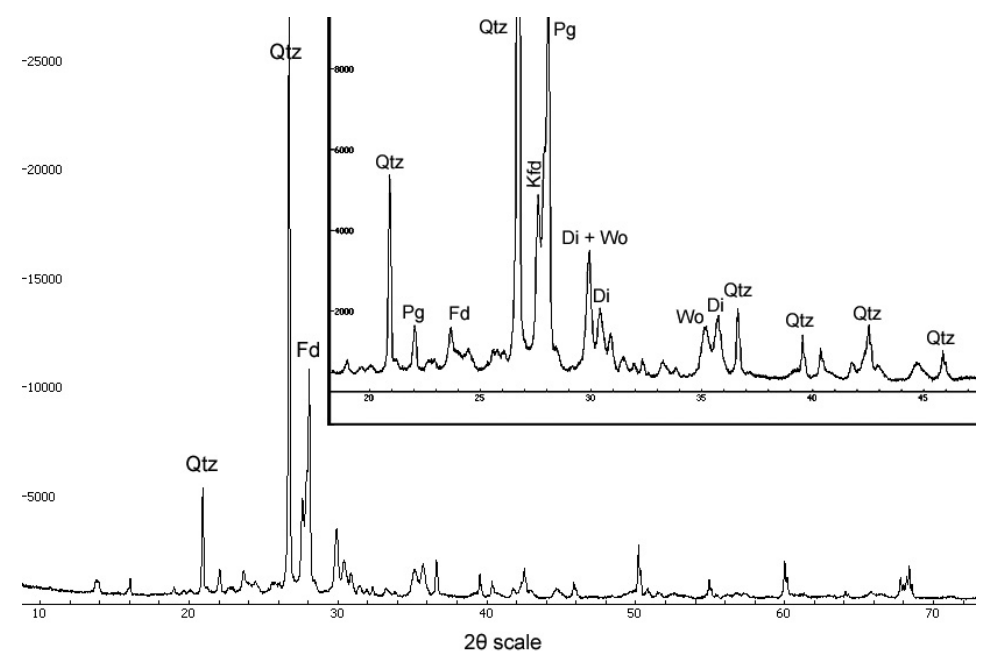

Figura 5 - Difractógrama de rayos $X$ de la muestra de cerámica calcárea DC-MT-03

Qtz: cuarzo; Fd: feldespato; Pg: plagioclasa; Kfd: feldespato potásico; Di: diópsido; Wo: wollastonita 


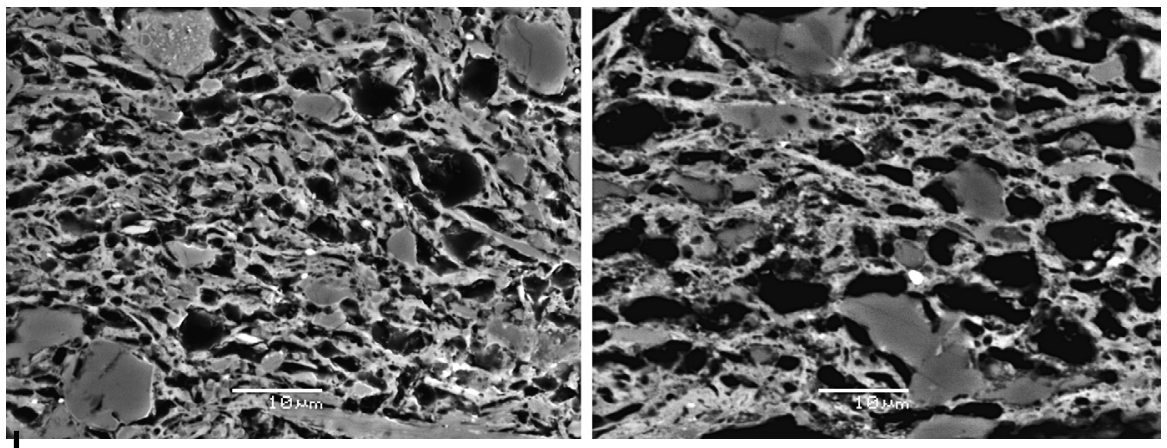

Figura 6 - Observaciones en MEB de la microestructura de las muestras de pasta blanca BR-MT-12 (izquierda) y DC-MT-03 (derecha)

Se nota una vitrificación extendida de la pasta con la presencia de poros finos

En cambio, las cerámicas de pasta roja y de pasta negra no presentan ninguna vitrificación de pasta y la presencia de granos de calcita primaria en ciertas muestras (DC-MT-02, BR-MT-04 et BR-MT-08) sugiere temperaturas de cocción inferiores a $700-750^{\circ} \mathrm{C}$.

Sobre las técnicas de decoración, aparte de las cerámicas calcáreas que tienen una pasta blanca, otros fragmentos presentan igualmente una superficie blanca (DC-MT-04, BR-MT-04 y BR-MT-08). En sección, se observa cómo este color es obtenido por la aplicación de un engobe blanco (fig. 7a y d) sobre el cual se puede aplicar una pintura roja para formar el motivo (fig. 7a). En el caso de la muestra DC-MT-03, en cambio, la pintura roja fue aplicada directamente sobre la pasta blanca, sin necesitar un engobe previo (fig. 7c). También existen decoraciones con motivos blancos sobre fondo rojo. En este caso, la pintura blanca se aplicó sobre una pasta roja (fig. $7 b$ ). Según los análisis de la composición química de estas capas de decoración, en el engobe blanco de la muestra BR-MT-08 y en la pintura blanca de la muestra BR-MT-11, se nota una fuerte proporción de silicio $\left(\mathrm{SiO}_{2}\right)$, aluminio $\left(\mathrm{Al}_{2} \mathrm{O}_{3}\right)$ y calcio $(\mathrm{CaO})$. La concentración de estos elementos sugiere, como en el caso de las pastas blancas, el uso de una arcilla calcárea. Al igual, el engobe blanco de la muestra DC-MT-04 tiene un contenido importante en estos elementos, pero se nota también una alta proporción de azufre (aprox. 10 \%). En este caso, es posible que el calcio y el azufre sean presentes en la forma de sulfatos de calcio, lo cual podría explicarse por la presencia de yeso $\left(\mathrm{CaSO}_{4}\right)^{5}$. El uso de este material es conocido como carga en las pinturas murales mochicas de la huaca de la Luna, en el valle de Moche (V. Wright, comunicación personal). Las pinturas rojas de las muestras BR-MT-08 y DC-MT-03 se caracterizan por concentraciones de calcio $(\mathrm{CaO})$ mucho más bajas que en los engobes $(<10 \%)$, y por porcentajes de alcalinos $\left(\mathrm{Na}_{2} \mathrm{O}\right.$ et $\left.\mathrm{K}_{2} \mathrm{O}\right)$ más altos. En la pintura de la muestra DC-MT-03, este porcentaje sobrepasa $10 \%$ de $\mathrm{CaO}$.

5 Los análisis de este engobe por espectrometría Raman permitirán determinar si se trata o no de sulfatos de calcio y, en caso afirmativo, de qué naturaleza son. 

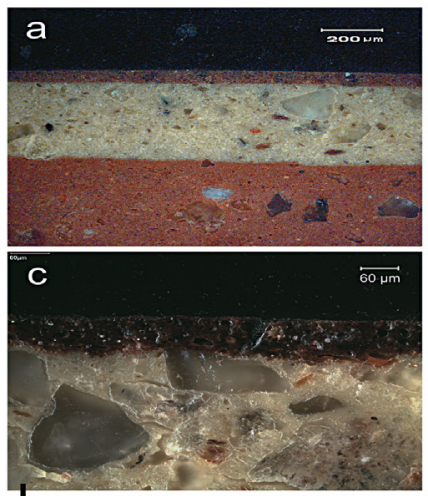

\begin{tabular}{|c|c|c|}
\hline & Engobe & Pintura \\
\hline $\mathrm{SiO}_{2}$ & 50.51 & 61.02 \\
\hline $\mathrm{Al} 2 \mathrm{O} 3$ & 15.24 & 19.34 \\
\hline $\mathrm{Fe} 2 \mathrm{O} 3$ & 7.17 & 7.90 \\
\hline $\mathrm{CaO}$ & 22.12 & 2.62 \\
\hline $\mathrm{MgO}$ & 1.95 & 1.45 \\
\hline $\mathrm{Na} 2 \mathrm{O}$ & 1.03 & 1.90 \\
\hline $\mathrm{K} 2 \mathrm{O}$ & 0.68 & 5.20 \\
\hline $\mathrm{TiO}_{2}$ & 0.61 & 0.57 \\
\hline $\mathrm{P} 2 \mathrm{O} 5$ & 0.38 & n.d. \\
\hline s & 0.32 & n.d. \\
\hline
\end{tabular}

\begin{tabular}{|lr|}
\hline $\mathrm{SiO} 2$ & Pintura \\
$\mathrm{Al} 2 \mathrm{O} 3$ & 21.74 \\
$\mathrm{Fe} 2 \mathrm{O} 3$ & 9.55 \\
$\mathrm{CaO}$ & 3.31 \\
$\mathrm{MgO}$ & 1.61 \\
$\mathrm{Na} 2 \mathrm{O}$ & 5.79 \\
$\mathrm{~K} 2 \mathrm{O}$ & 4.85 \\
$\mathrm{TiO} 2$ & 0.90 \\
$\mathrm{P} 2 \mathrm{O} 5$ & 0.23 \\
$\mathrm{~S}$ & n.d. \\
\hline
\end{tabular}
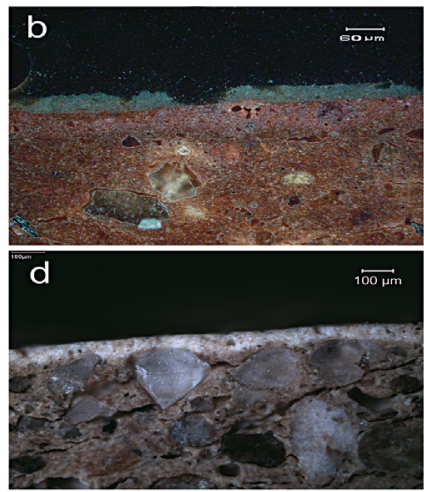

\begin{tabular}{|c|c|}
\hline $\mathrm{SiO}_{2}$ & $\begin{array}{r}\text { Pintura } \\
37.06\end{array}$ \\
\hline $\mathrm{Al} 2 \mathrm{O} 3$ & 12.50 \\
\hline $\mathrm{Fe} 2 \mathrm{O} 3$ & 4.36 \\
\hline $\mathrm{CaO}$ & 36.23 \\
\hline $\mathrm{MgO}$ & 2.26 \\
\hline $\mathrm{Na} 2 \mathrm{O}$ & 2.34 \\
\hline $\mathrm{K} 2 \mathrm{O}$ & 1.23 \\
\hline $\mathrm{TiO} 2$ & 0.43 \\
\hline P2O5 & n.d. \\
\hline $\mathrm{s}$ & 2.24 \\
\hline $\mathrm{SiO} 2$ & $\begin{array}{r}\text { Engobe } \\
39.37\end{array}$ \\
\hline $\mathrm{Al} 2 \mathrm{O} 3$ & 11.75 \\
\hline $\mathrm{Fe} 2 \mathrm{O} 3$ & 3.25 \\
\hline $\mathrm{CaO}$ & 27.45 \\
\hline $\mathrm{MgO}$ & 4.25 \\
\hline $\mathrm{Na} 2 \mathrm{O}$ & 1.25 \\
\hline $\mathrm{K} 2 \mathrm{O}$ & 1.73 \\
\hline $\mathrm{TiO} 2$ & 0.26 \\
\hline P2O5 & n.d. \\
\hline & 10.51 \\
\hline
\end{tabular}

Figura 7 - Observaciones en sección de diferentes técnicas de decoración de cerámicas de Dos Cabezas y El Brujo

a: BR-MT-08. Pintura roja sobre engobe blanco; b: BR-MT-11. Pintura blanca sobre pasta roja; c: DC-MT-03. Pintura roja sobre pasta blanca; d: DC-MT-04. Engobe blanco sobre pasta rosada

En cuanto a las cerámicas Línea Fina de San José de Moro y Pacatnamú, presentan, en sección, colores de pasta bastante características de este grupo, con un margen interno de color gris y un margen externo de color rojo a anaranjado (fig. 8). Estos colores son característicos de una cocción en atmósfera reductora seguida de un enfriamiento rápido en atmósfera oxidante (Rye, 1981: 115-117). Se trata de vasijas de forma muy cerrada, lo que favorece un fenómeno de oxidación que se produce desde la pared externa hacia la pared interna, y no desde las paredes internas y externas hacia el centro como en el caso de las vasijas de forma abierta (Picon, 1973).

También se nota que este grado de oxidación varía de una muestra a otra. Estas diferencias son relacionadas con la velocidad de enfriamiento de las vasijas después de la cocción. Cuanto más rápido es el enfriamiento, más delgada es la zona oxidada. Según Rye (1981: 118), la presencia de esta zona oxidada adyacente a la superficie es característica de una cocción en área abierta o en pozo seguida de un enfriamiento muy rápido al aire libre.

Las observaciones de la microestructura de estas muestras en MEB revelan diferentes grados de vitrificación, que se extienden de una vitrificación inicial hasta
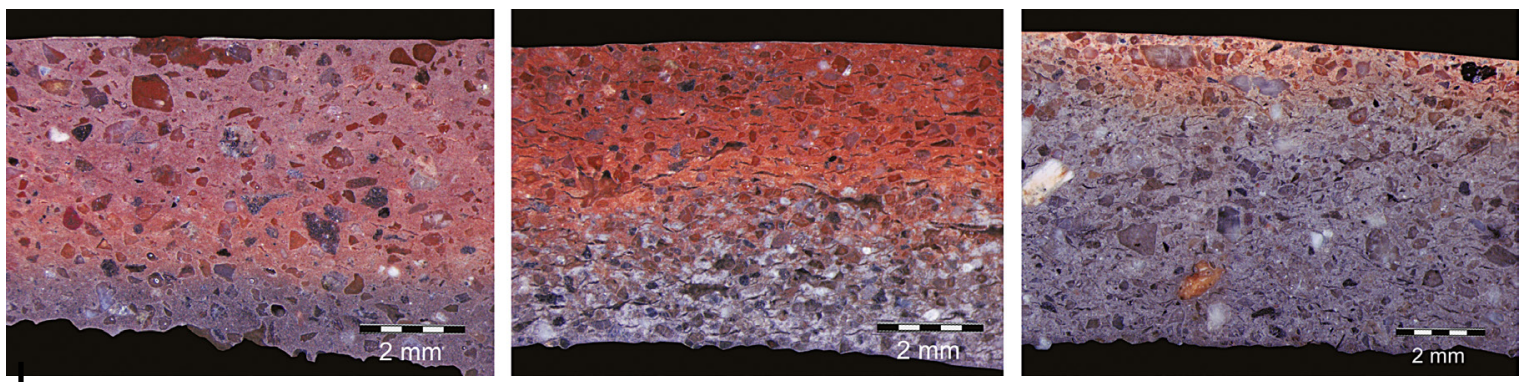

Figura 8 - Observaciones en microscopia óptica de las secciones de vasijas Línea Fina de San José de Moro 
una vitrificación continua con la formación de poros finos a medios (fig. 9). Estas diferentes microestructuras indican temperaturas de cocción que se escalonan entre $750-800^{\circ} \mathrm{C}$ (vitrificación inicial) y $900-1000^{\circ} \mathrm{C}$ (vitrificación continua con poros medios) (Maniatis \& Tite, 1981).
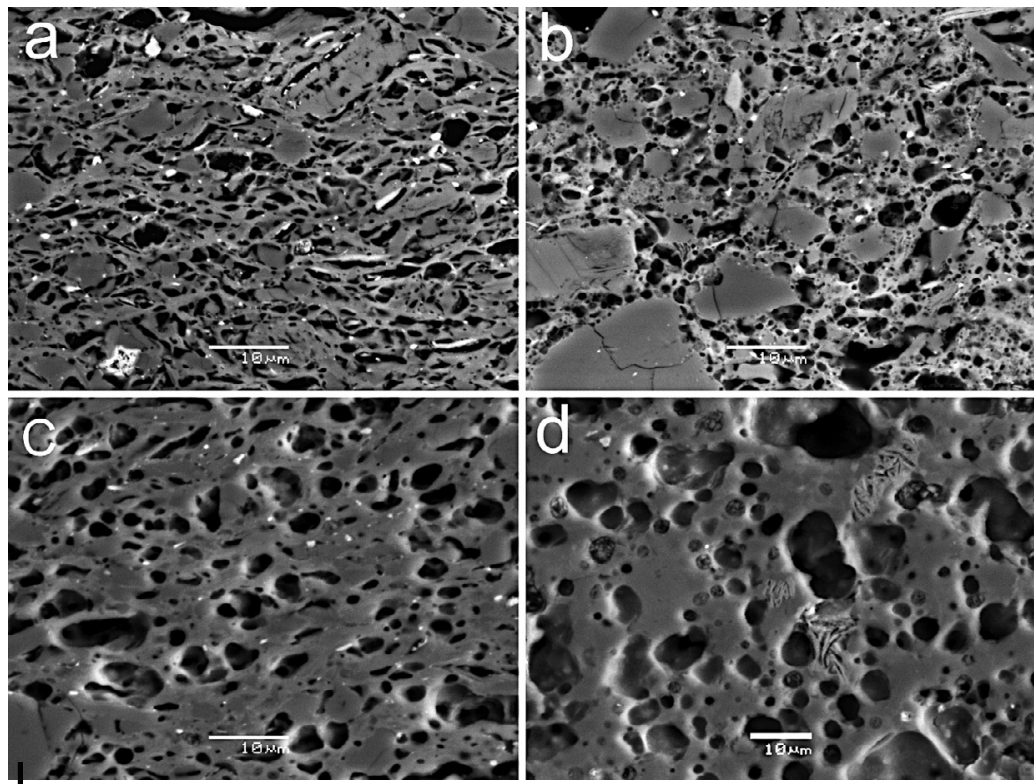

Figura 9 - Observaciones en MEB (imágenes BSE) de la microestructura de cerámicas Línea Fina de San José de Moro (a, b y c) y Pacatnamú (d)

a: Vitrificación extendida; b: Vitrificación continua con poros finos; c: Vitrificación continua con poros finos; d: Vitrificación continua con poros medios

Sobre las técnicas de decoración, las vasijas Línea Fina son sistemáticamente cubiertas de un engobe blanco, sobre el cual se aplica una pintura de color rojo a negro (fig. 10). Los primeros datos que tenemos sobre la naturaleza de estos engobes indican altas concentraciones de silicio $\left(\mathrm{SiO}_{2}\right)$, aluminio $\left(\mathrm{Al}_{2} \mathrm{O}_{3}\right)$ y calcio $(\mathrm{CaO})$, lo que sugiere, como en el caso de las muestras más tempranas, el uso de arcillas calcáreas para su elaboración. En cuanto a las pinturas que forman el motivo de la decoración, se componen principalmente de silicio $\left(\mathrm{SiO}_{2}\right)$, aluminio $\left(\mathrm{Al}_{2} \mathrm{O}_{3}\right)$ y hierro $\left(\mathrm{Fe}_{2} \mathrm{O}_{3}\right)$ (fig. 11). Esta composición evidencia el uso de óxidos de hierro como pigmento, y su probable mezcla con una arcilla muy diluida (fuerte proporción de silicio y aluminio). Según los análisis efectuados por Chapdelaine et al. (1997), el mismo proceso se empleaba en la preparación de engobes blancos y rojos de cerámicas de la fase IV procedentes del sitio huacas de Moche. 

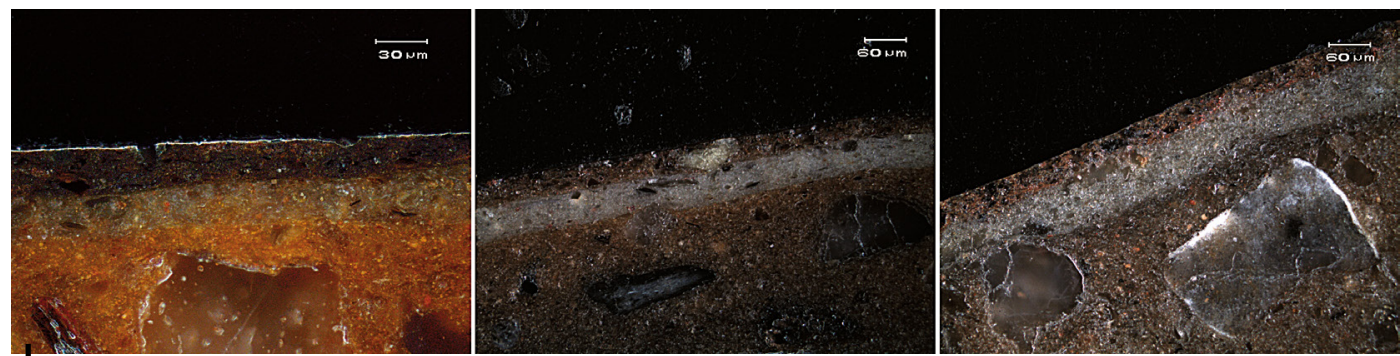

Figura 10 - Observaciones en sección de las técnicas de decoración de cerámicas Línea Fina de San José de Moro

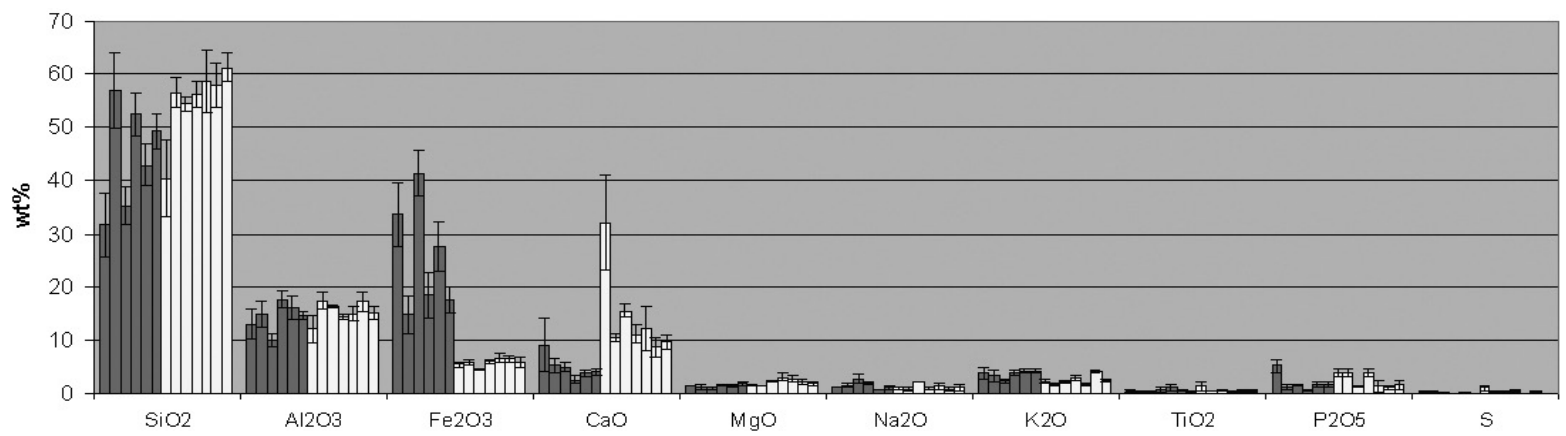

Figura 11 - Histograma de composición elemental (\% de óxidos) de las decoraciones de cerámicas Línea Fina de San José de Moro

Los histogramas blancos corresponden a los engobes y los histogramas grises a las pinturas

\section{DISCUSIÓN Y PERSPECTIVAS}

La proporción de temperante observada en las cerámicas de este estudio (> 20 \%) y su naturaleza (gran cantidad de cuarzos) sugieren la incorporación de arena a la arcilla durante la etapa de preparación de la pasta. Este añadido no solo permite facilitar el secado de las piezas, sino que también les confiere más dureza. Al contrario, disminuye la resistencia mecánica de las vasijas (Tite et al., 2001: 307). La fineza de las paredes de las vasijas de este estudio $(<10 \mathrm{~mm})$ también contribuye a la disminución de su resistencia mecánica. En cambio, una pared delgada aumenta la resistencia al choque térmico por el hecho de que los gradientes de temperatura a través de la pared y, entonces, las tensiones que conducen al inicio de fisuras, son reducidos (Tite et al., 2001: 319). Esta resistencia al choque térmico también depende por mucho de la proporción y de la naturaleza del temperante empleado. Como lo demuestran los experimentos de Kilikoglou et al. (1995), una proporción de cuarzo superior al 20 \% permite aumentar la disipación de energía al momento de la propagación de fisuras, y entonces la resistencia al choque 
térmico. Pero si la proporción de este mineral es demasiado importante presenta un riesgo al momento de la subida de temperatura debido a su fuerte coeficiente de dilatación térmica (Rye, 1976: 116-117). Los trabajos de Kilikoglou et al. indican que para lograr una buena resistencia al choque térmico la proporción óptima de cuarzo se sitúa alrededor del 20 \% en volumen. Aun si la proporción exacta de granos de cuarzo en las muestras de este estudio todavía no ha sido determinada, las primeras observaciones de secciones delgadas en microscopia polarizante señalan que esta proporción no se aleja mucho de la indicada por estos autores. En el caso de las cerámicas Línea Fina, estos datos coinciden con el modo de cocción que se puede inferir de los colores de pasta en sección. Estas cerámicas presentan una coloración típica de una cocción en área abierta o en pozo seguida de un enfriamiento rápido al aire libre. Ahora bien, estos procesos de cocción implican subidas de temperatura muy rápidas. En una cocción en área abierta, el tiempo medio para alcanzar la temperatura máxima es de 22 min, y de 41 min en una cocción en pozo (Gosselain, 1992: 246). Entonces, las vasijas cocidas de este modo deben poder soportar semejantes variaciones térmicas. La presencia de estructuras de cocción en pozo en varios talleres de producción cerámica mochica de las fases IV y $\mathrm{V}$ evidencia el uso de este tipo de cocción. Por otra parte, los diferentes grados de vitrificación observados en las vasijas Línea Fina indican temperaturas de cocción bastante variadas (de $750-800^{\circ} \mathrm{C}$ hasta 900-1000 C), lo que parece coincidir con el hecho de que durante una cocción en pozo, es muy difícil controlar con precisión las temperaturas y estas pueden variar considerablemente entre diferentes puntos de la estructura de cocción (Gosselain, 1992: 256). Las medidas termométricas efectuadas por Salazar et al. (1993) durante una cocción en pozo en el pueblo de Mórrope6 (departamento de Lambayeque) evidencian, dentro de una misma cocción, temperaturas que se escalonan entre $498^{\circ} \mathrm{C}$ y $1032^{\circ} \mathrm{C}$, con un promedio de $870^{\circ} \mathrm{C}$ al nivel de las vasijas colocadas en el fondo del pozo (sobre la cama de leña) y un promedio de $910^{\circ} \mathrm{C}$ al nivel de las vasijas de la hilera superior.

En el caso de las cerámicas calcáreas, en cambio, no parece que tal proceso haya sido posible. Como lo hemos visto, la cocción de estas vasijas necesita un mejor control de las temperaturas. El análisis de los dos fragmentos de pasta blanca sugiere temperaturas de cocción superiores a $950^{\circ} \mathrm{C}$. Ahora bien, es difícil alcanzar semejantes temperaturas y mantenerlas con una cocción en área abierta o en pozo (que las vasijas sean separadas del combustible por tiestos o no). Solo las cocciones en horno permiten tal control. Hasta la fecha, el único horno de alfarero conocido para el periodo mochica es el de Cerro Mayal, que funcionó durante la fase Mochica IV. Para los periodos más tempranos, no disponemos de información sobre las estructuras de cocción empleadas.

Además de la información que nos proporcionan sobre las técnicas de cocción, las vasijas de pasta blanca también son muy interesantes tratándose de la explotación

6 El combustible usado durante esta cocción fue leña de algarrobo y de vichayo, estiércol de vaca y de cabra y horajascas y ramas pequeñas de algarrobo (Salazar et al., 1993 : 689) 
y del origen de las materias primas. Algunos autores se refieren a la materia prima empleada en la elaboración de estas vasijas y de los engobes blancos y mencionan el uso de una «arcilla blanca» (Donnan \& McClelland, 1999: 28; Donnan, 1992; Chapdelaine et al., 1995: 197). Esta denominación parece hacer referencia a las arcillas caoliníticas, cuyos principales yacimientos se localizan en la sierra. Este alejamiento entre los yacimientos de caolín y el territorio costanero ocupado por los mochicas ha llevado a ciertos arqueólogos a considerar las cerámicas de pasta blanca de esta cultura como una prueba de la existencia de redes de intercambios con culturas andinas (Chapdelaine et al., 1995: 243; Donnan \& McClelland, 1999: 305). Pero los análisis que hemos efectuado en este estudio demuestran que las cerámicas de pasta blanca y los engobes blancos son mayormente elaborados a partir de arcillas calcáreas y no de arcillas caoliníticas. Es importante mencionar que las margas (arcillas calcáreas) y las calizas son abundantes en la costa norte peruana. Se encuentran especialmente en formaciones del Cretácico Medio (Pariatambo y Chulec) y del Jurásico Superior (Chicama)7. Entonces, las materias primas empleadas por los mochicas pueden ser de origen local. Si las cerámicas de pasta blanca son escasas en la producción mochica, no es por la escasez de la materia prima empleada para su fabricación. En cambio, el hecho de que estas cerámicas necesiten una cocción a alta temperatura (superiora a 950 $\mathrm{C}$ ) podría constituir una cierta dificultad para los alfareros mochicas quienes, aparentemente, usaban más la cocción en pozo. Sin embargo, la cultura Mochica produjo también numerosos objetos de cobre, cuya temperatura de fusión es de $1084^{\circ}$ C. Esto demuestra que los artesanos de esta sociedad controlaban perfectamente la obtención de altas temperaturas. Además, la presencia de un sector agrupando diferentes artesanías en el sitio de las huacas de Moche y el carácter compuesto de ciertos objetos producidos por esta sociedad, como las piezas de cerámica o de metal con incrustaciones de piedra, concha o hueso, parecen indicar que estas diferentes artesanías no eran compartimentadas según el tipo de material trabajado. Entonces, es difícil pensar que solamente los artesanos metalurgistas eran capaces de lograr altas temperaturas, mientras que los alfareros no lo podían. Otra explicación de esta rareza de las cerámicas de pasta blanca podría ser la cantidad de combustible que requiere la cocción de estas vasijas. La aplicación de engobes blancos permite usar temperaturas de cocción más bajas y, requiere, por lo tanto, menos combustible.

Finalmente, el estudio de las técnicas de decoración señala que varias soluciones técnicas podían ser empleadas para obtener un resultado similar. Las decoraciones rojas sobre un fondo blanco, que caracterizan gran parte de la cerámica fina mochica, podían ser obtenidas por la aplicación de un engobe blanco y luego de una pintura roja, o por la aplicación de una pintura roja directamente sobre la superficie de una vasija de pasta blanca. Es interesante constatar que Carole Fraresso hizo las mismas observaciones acerca de las técnicas de dorado empleadas en la fabricación de objetos de metal (Fraresso, 2007). Podemos interrogarnos, 
como lo hace esta autora, sobre los motivos de estas elecciones técnicas. ¿Están relacionadas con la existencia de prácticas técnicas regionales, o incluso locales? ¿Pueden ser estas prácticas el reflejo de la existencia de diferentes grupos de artesanos o de diferentes «territorios técnicos»?

Por supuesto, el estado actual de las investigaciones sobre las técnicas artesanales mochicas no permite responder a estos interrogantes. Sin embargo, estas investigaciones demuestran la importancia de tomar en cuenta consideraciones de orden técnico en la definición de la organización de los diferentes grupos humanos que formaban la sociedad Mochica.

\section{Agradecimientos}

Quiero agradecer a las personas que hicieron posible este trabajo de investigación. En primer lugar, a Luis Jaime Castillo por haberme dado acceso al material cerámico del Proyecto Arqueológico San José de Moro (PASJM) y por el apoyo que siempre ha brindado a este trabajo de investigación. También quiero agradecer a toda la gente del PASJM por su enseñanza y ánimo a lo largo de las temporadas de excavación que tuve suerte de pasar con ellos. A Christopher Donnan por haberme facilitado el acceso al material de Dos Cabezas y Pacatnamú. A Régulo Franco por haberme confiado material del Proyecto Arqueológico Complejo El Brujo y a Carmen Gamarra de la Cruz por haberme asistido en la selección de muestras.

\section{Referencias citadas}

ANDERS, M., CHANG, V., TOKUDA, L., QUIROZ, S. \& SHIMADA, I., 1994 - Producción cerámica del Horizonte Medio Temprano en Maymi, Valle de Pisco, Perú. In: Tecnología y organización de la producción de cerámica prehispánica en los Andes (I. Shimada, ed.): 249-267; Lima: Pontificia Universidad Católica del Perú (PUCP) Fondo Editorial.

ARMAS, J., CHAMORRO, V. \& JARA, G., 1993 - Investigaciones arqueológicas en el Complejo Huaca del Sol y La Luna: talleres alfareros de la sociedad Moche; Trujillo: Universidad Nacional de Trujillo, Escuela de Arqueología. Informe de Prácticas Preprofesionales.

ARNOLD, D. E., 1972 - Mineral analysis of ceramic materials from Quinua, Department of Ayacucho, Peru. Archaeometry, 14 (1): 93-102.

ARNOLD, D. E., 1975 - Ceramic ecology of the Ayacucho Basin, Peru: Implications for Prehistory. Current Anthropology, 16 (2): 183-194.

ARNOLD, D. E., 1985 - Ceramic Theory and Cultural Process, 284 pp.; New-York: Cambridge University Press.

ATTARIAN, C. J., 1996 - Plant Foods and Ceramic Production: A Case Study of Mochica Ceramic Production Specialists in the Chicama Valley, Peru; Los Angeles: University of California, Department of Anthropology. Tesis de Maestría.

BANKES, G., 1985 - The manufacture and circulation of paddle and anvil pottery on the north coast of Peru. World Archaeology, 17: 269-276. 
BAWDEN, G., 1982 - Galindo: A study in cultural transition during the Middle Horizon. In: Chan Chan: Andean Desert City (M. E. Moseley \& K. Day, eds.): 285-320; Albuquerque: The University of New Mexico Press.

BAWDEN, G., 1996 - The Moche, 375 pp.; Oxford: Blackwell Publishers Ltd.

BERNIER, H., 2008 - Especialización artesanal en el sitio Huacas de Moche: contextos de producción y función sociopolítica. In: Arqueología Mochica: Nuevos Enfoques (L. J. Castillo, J. Rucabado, H. Bernier \& G. Lockard, eds.): 33-51; Lima: Pontificia Universidad Católica del Perú (PUCP)-Fondo Editorial, Instituto Francés de Estudios Andinos (IFEA). Actas del Primer Congreso Internacional de Jóvenes Investigadores sobre la Cultura Mochica (Lima, 4 y 5 de agosto de 2004).

CAMINO, L., 1982 - Los que vencieron el tiempo. Símbila costa norte. Perfil etnográfico de un centro alfarero, 139 pp.; Piura: Centro de Investigación y Promoción del Campesinado.

CAMINO, L., 1989 - Olleros y sogueros de Sondorillo, Piura. Boletín de Lima, 61: 25-28.

CASTILLO, L. J. \& DONNAN, C. B., 1994 - Los Mochicas del Norte y los Mochicas del Sur, una perspectiva desde el valle de Jequetepeque. In: Vicús (K. Makowski, ed.): 143181; Lima: Banco de Crédito del Perú, Colección Arte y Tesoros del Perú.

CASTILLO, L. J. \& UCEDA, S., 2008 - The Mochicas. In: Handbook of South America Archaeology (H. Silverman \& W. Isbell, eds.): 707-729; New-York: Springer.

CHAPDELAINE, C., KENNEDY, G. \& UCEDA, S., 1995 - Activación neutrónica en el estudio de la producción local de cerámica ritual en el sitio Huacas de Moche, Perú. Bulletin de I'Institut Français d'Études Andines, 24 (2): 183-212.

CHAPDELAINE, C., MINEAU, R., UCEDA, S., 1997 - Estudio de los pigmentos de la cerámica ceremonial Moche con ayuda de un microscopio electrónico de barrido. Bulletin de I'Institut Français d'Études Andines, 26 (2): 229-245.

CHAPDELAINE, C., KENNEDY, G., UCEDA, S., 2001 - Neutron activation analysis of metal artefacts from the Moche site, north coast of Peru. Archaeometry, Vol. 43 (3): 373391.

DeMARRAIS, E., CASTILLO, L. J. \& EARLE, T., 1996 - Ideology, Materialization, and Power Strategies. Current Anthropology, 37 (1): 15-31.

DONNAN, C. B., 1971 - Ancient Peruvian potters' marks and their interpretation through ethnographic analogy. American Antiquity, 36 (4): 460-466.

DONNAN, C. B., 1992 - Ceramics of Ancient Peru, 128 pp.; Los Angeles: University of California Fowler, Museum of Cultural History.

DONNAN, C. B., 2004 - Moche Portraits from Ancient Peru, 220 pp.; Austin: University of Texas Press.

DONNAN, C. B. \& McCLELLAND, D., 1999 - Moche Fineline Painting. Its Evolution and its artists, 319 pp.; Los Angeles: UCLA Fowler Museum of Cultural History.

DRUC, I., 1996 - De la etnografía hacia la arqueología: aportes de entrevistas con ceramistas de Ancash (Perú) para la caracterización de la cerámica prehispánica. Bulletin de l'Institut Français d'Études Andines, 25 (1): 17-41.

DRUC, I., 2000 - ¿Shashal o no shashal? Esa es la cuestión. Etnoarqueología cerámica en la zona de Huari, Ancash. Bulletin de I'Institut Français d'Études Andines, 30 (1): 157-173.

ECHALLIER, J.-C., 1984 - Éléments de technologie céramique et d'analyse des terres cuites archéologiques. Documents d'archéologie méridionale, Méthodes et techniques 3: 5-40. 
FRARESSO, C., 2007 - L'usage du métal dans la parure et les rites de la culture Mochica, Pérou (150-850 ap. J. -C.); Burdeos: Université Michel de Montaigne Bordeaux 3. Tesis de doctorado, «Spécialité Physique des Archéomatériaux».

GOSSELAIN, O., 1992 - Bonfire of the enquiries. Pottery firing temperatures in Archaeology: What for? Journal of Archaeological Science, 19 (3): 243-259.

KILIKOGLOU, V., VEKINIS, G. \& MANIATIS, Y., 1995 - Toughening of ceramic earthenwares by quartz inclusions: an ancient art revisited. Acta Metallurgica et Materialia, 43 (8): 2959-2965.

LARCO HOYLE, R., 1948 - Cronología arqueológica del norte del Perú. Biblioteca del Museo Arqueológico Rafael Larco Herrera, Hacienda Chiclín. Buenos Aires: Sociedad Geográfica Americana. [Reimpreso en: Arqueológicas, 2001, vol. 25; Lima].

LAVALLÉE, D., 1967 - La poterie de Aco (Andes centrales du Pérou). Objets et Mondes, T. VII, 2: 103-120.

LITTO, G., 1976 - South American Folk Pottery. Traditional techniques from Peru, Ecuador, Bolivia, Venezuela, Chile, Colombia, 223 pp.; New-York: Watson-Guptill Publications.

MAGGETTI, M., 1982 - Phase Analysis and its Significance for Technology and Origin. In: Archaeological Ceramics (J. S. Olin \& A.D. Franklin, eds.): 121-133; Washington: Smithsonian Institution.

MANIATIS, Y. \& TITE, M. S., 1981 - Technological examination of Neolithic Bronze Age pottery from Central and Southeast Europe and from the Near East. Journal of Archaeological Science, 8 (1): 59-76.

MONZON, S., 1991 - El estudio de la cerámica y su contribución a una investigación interregional - El caso de Piura. Bulletin de l'Institut Français d'Études Andines, 20 (2): 589-597.

MOUTARDE, F., 2006 - L'évolution du couvert ligneux et de son exploitation par l'homme dans la vallée du Lurin (côte centrale du Pérou), de l'Horizon Ancien (900-100 av. J.-C.) à I'Horizon Tardif (1460-1532 ap. J.-C.). Approche anthracologique. Volume II: Atlas anthracologique; París: Universidad Paris I, Panthéon-Sorbonne. Tesis de doctorado, Especialidad «Archéologie et Environnement».

PEREGRINE, P., 1991 - Some political aspects of craft specialization. World Archaeology, 23 (1): 1-11.

PÉRINET, G., 1960 - Contribution de la diffraction des rayons X à l'évaluation de la température de cuisson d'une céramique. VIIth International Ceramic Congress; Londres.

PETERS, T. \& IBERG, R., 1978 - Mineralogical changes during firing of calcium-rich brick clays. American Ceramic Society Bulletin, 57 (5): 503-509.

PICON, M., 1973 - Introduction à l'étude technique des céramiques sigillées de Lezoux, 135 pp.; Dijon: Centre de Recherches sur les techniques gréco-romaines.

PICON, M., 1992 - Ethnoarchéologie et recherches en laboratoire : le cas des techniques céramiques. In: Ethnoarchéologie : justification, problèmes, limites (F. Audouze, ed.): 115-126; Juan-les-Pins: Éditions APDCA. Actes des XIIlèmes Rencontres Internationales d'Archéologie et d'Histoire d'Antibes, 17-19 octobre 1991.

POZZI-ESCOT, D., ALARCÓN, M. G. \& VIVANCO, C., 1993 - Instrumentos de alfareros de la época Wari. Bulletin de I'Institut Français d'Études Andines, 22 (2): 467-496.

RAMÓN JOFFRÉ, G., 1999 - Producción alfarera en Santo Domingo de los Olleros (Huarochirí - Lima). Bulletin de l'Institut Français d'Études Andines, 28 (2): 215-248. 
RAMÓN JOFFRÉ, G., 2008 - Producción alfarera en Piura (Perú): estilos técnicos y diacronía. Bulletin de I'Institut Français d'Études Andines, 37 (3): 477-509.

RAVINES, R. (ed.), 1978 - Tecnología Andina, 821 pp.; Lima: Instituto de Estudios Peruanos (IEP).

RAVINES, R., 1989 - Principales comunidades y centros alfareros del Perú. In: La cerámica tradicional del Perú (R. Ravines \& F. Villiger, eds.): 45-57; Lima: Los Pinos.

RENGIFO CHUNGA, C. \& ROJAS VEGA, C., 2008 - Talleres especializados en el conjunto arqueológico Huacas de Moche: el carácter de los especialistas y su producción. In: Arqueología Mochica: Nuevos Enfoques (L. J. Castillo, J. Rucabado, H. Bernier \& G. Lockard, eds.): 325-339; Lima: Pontificia Universidad Católica del Perú (PUCP)Fondo Editorial, Instituto Francés de Estudios Andinos (IFEA). Actas del Primer Congreso Internacional de Jóvenes Investigadores sobre la Cultura Mochica (Lima, 4 y 5 de agosto del 2004.

RUSSEL, G. S., LEONARD, B. L. \& BRICEÑO ROSARIO, J., 1994 - Producción de cerámica Moche a gran escala en el valle de Chicama, Perú: el taller de Cerro Mayal. In: Tecnología y organización de la producción de cerámica prehispánica en los Andes (I. Shimada, ed.): 201-227; Lima: Pontificia Universidad Católica del Perú (PUCP)Fondo Editorial.

RUSSELL, G. S. \& JACKSON, M. A., 2001 - Political economy and patronage at Cerro Mayal, Peru. In: Moche Art and Archaeology in Ancient Peru (J. Pillsbury, ed.): 159175; Washington: National Gallery of Art, Studies in the History of Art 63, Center for Advanced Studies in the Visual Arts.

RYE, O., 1976 - Keeping your temper under control: materials and the manufacture of Papuan pottery. Archaeology and Physical Anthropology in Oceania, 11 (2): 106137.

RYE, O., 1981 - Pottery Technology: Principles and Reconstruction; Washington, D.C.: Taraxacum Inc..

SABOGAL WIESSE, J. R., 1982 - La cerámica de Piura, 263 pp.; Quito: Instituto Andino de Artes Populares.

SALAZAR RODRÍGUEZ, J., LÓPEZ CARRANZA, E. \& VREELAND, J. M., 1993 - Medidas de las temperaturas de un horno artesanal de Mórrope, departamento de Lambayeque, utilizado para la fabricación tradicional de alfarería. Bulletin de I'Institut Français d'Études Andines, 22 (3): 685-699.

SHIMADA, I., 1985 - Perception, Procurement and Management of Resources: Archaeological Perspective. In: Andean Ecology and Civilization (S. Masuda, I. Shimada \& C. Morris, eds.): 357-400; Tokyo: University of Tokyo Press.

SHIMADA, I., 1994a - Pampa Grande and the Mochica Culture, 341 pp.; Austin: University of Texas Press.

SHIMADA, I., 1994b - La producción de cerámica en Mórrope, Perú: productividad, especialización y espacio vistos como recursos. In: Tecnología y organización de la producción de cerámica prehispánica en los Andes (I. Shimada, ed.): 295-319; Lima: Pontificia Universidad Católica del Perú (PUCP)-Fondo Editorial.

SHIMADA, I., ELERA, C., CHANG, V., NEFF, H., GLASCOCK, M., WAGNER, U. \& GEBHARD, R., 1994 - Hornos y producción de cerámica durante el periodo formativo en Batán Grande, costa norte del Perú. In: Tecnología y organización de la producción de cerámica prehispánica en los Andes (I. Shimada, ed.): 67-115; Lima: Pontificia Universidad Católica del Perú (PUCP)-Fondo Editorial. 
TITE, M. S., 1999 - Pottery production, distribution and consumption. The contribution of the physical sciences. Journal of Archaeological Method and Theory, 6 (3): 181-233.

TITE, M. S., KILIKOGLOU, V. \& VEKINIS, G., 2001 - Strength, toughness and thermal shock resistance of ancient ceramics, and their influence on technological choice. Archaeometry, 43 (3): 301-324.

TOPIC, T. L., 1977 - Excavations at Moche; Cambridge: Harvard University, Department of Anthropology. PhD Dissertation.

TSCHOPIK Jr., H. 1950 - An Andean ceramic tradition in historical perspective. American Antiquity, 15 (3): 196-218.

UCEDA, S. \& ARMAS, J., 1997 - Los talleres alfareros en el centro urbano Moche. In: Informe de las Investigaciones en la Huaca de la Luna 1995 (S. Uceda, E. Mujica \& R. Morales, eds.): 93-104; Trujillo: Universidad Nacional de Trujillo, Facultad de Ciencias Sociales.

WILSON, D. J., 1988 - Prehispanic settlement patterns in the Lower Santa Valley Peru, 540 pp.; Washington: Smithsonian Institution Press. 